\title{
Effects of Magnetic Field and Thermal Radiation on Stagnation Flow and Heat Transfer of a Power-Law Fluid over a Shrinking Sheet
}

\author{
Samir Kumar Nandy \\ Department of Mathematics, A.K.P.C Mahavidyalaya, Bengai, Hooghly 712 611, India \\ Correspondence should be addressed to Samir Kumar Nandy; nandysamir@yahoo.com
}

Received 29 June 2013; Accepted 23 September 2013

Academic Editor: Francisco Chinesta

Copyright (c) 2013 Samir Kumar Nandy. This is an open access article distributed under the Creative Commons Attribution License, which permits unrestricted use, distribution, and reproduction in any medium, provided the original work is properly cited.

\begin{abstract}
An analysis is made on the steady two-dimensional boundary layer magnetohydrodynamic (MHD) stagnation-point flow and radiative heat transfer of an electrically conducting power-law fluid over a shrinking sheet which is shrunk in its own plane with a velocity proportional to the distance from a fixed point. The similarity transformations are used to transform the boundary layer equations into a system of nonlinear ordinary differential equations which are then solved numerically using shooting technique. It is found that multiple solutions exist for a certain range of the ratio of the shrinking velocity to the free stream velocity (i.e., $\alpha$ ) which again depends on the magnetic parameter $(M)$ and the power-law index parameter $(n)$. The results pertaining to the present study indicate that as the strength of the magnetic parameter increases, the range of $\alpha$ where similarity solutions exist gradually increases. It is also observed that the temperature at a point decreases with increase in $M$ for the first solution branch, whereas it increases with increase in $M$ for the second solution branch. The reported results are in good agreement with the available published work in the literature.
\end{abstract}

\section{Introduction}

From a technological point of view, non-linear fluid rheology is of special interest and has practical applications. Hence the study of non-Newtonian fluid is very much important. A large number of industrial fluids such as molten plastics, artificial fibres, food stuff, polymers, and slurries are nonNewtonian in their flow characteristics. Many of the inelastic non-Newtonian fluids encountered in chemical engineering processes and biochemical industries are known to follow the empirical Ostwald-de Waele model (see Metzner [1]) or socalled power-law model. This model is described by a simple nonlinear equation of state for inelastic fluids which includes linear Newtonian fluids as a special case.

Also, the study of magnetohydrodynamic (MHD) flow of an electrically conducting fluid is of considerable interest in modern metallurgical and metal-working processes. This type of flow has attracted the interest of many researchers due to its application in many engineering problems such as MHD generators, plasma studies, nuclear reactors, and geothermal energy extractions. Also by the application of magnetic field, hydromagnetic techniques are used for the purification of molten metals from nonmetallic inclusions. Therefore the type of problem that we are dealing with is very useful to polymer technology and metallurgy.

Stagnation-point flow is a topic of significance in fluid mechanics as it appears in virtually all flow fields of science and engineering. In some cases flow is stagnated by a solid wall, while in others a free stagnation point or a line exists in the interior of the fluid domain. Hiemenz [2] was the first to discover that the stagnation-point flow can be analyzed by the Navier-Stokes equations for the two-dimensional case. The axisymmetric case was studied by Homann [3]. Thereafter numerous investigations were made on the stretching sheet problem with linear stretching in different directions [4-16].

Recently, the boundary layer flow due to a shrinking sheet has attracted considerable interest. One of the common applications of shrinking sheet problems in industries and engineering is shrinking film. In packaging of bulk products, shrinking film is very useful as it can be unwrapped easily 
with adequate heat. Shrinking problem can also be applied to study the capillary effects in small pores, the shrinking-swell behaviour, and the hydraulic properties of agricultural clay soils. The associated changes in hydraulic and mechanical properties of such soils will seriously hamper predictions of the flow and transport processes which are essential for agricultural development and environmental strategies.

For this flow configuration, the fluid is attracted towards a slot, and the flow is quite different from the stretching case. From a physical point of view, vorticity generated at the shrinking sheet is not confined within a boundary layer, and a steady flow is not possible unless either a stagnationpoint flow towards the sheet is applied, or adequate suction is applied at the sheet. This type of shrinking flow is essentially a backward flow as discussed by Goldstein [17]. For a backward flow configuration, the fluid losses memory of the perturbation introduced by the slot. As a result, the flow induced by the shrinking sheet shows quite distinct physical phenomena from the stretching case.

Miklavcic and Wang [18] investigated both two-dimensional and axisymmetric viscous flow induced by a shrinking sheet in the presence of uniform suction. The above shrinking sheet problem with a power-law surface velocity was analyzed by Fang [19]. The unsteady viscous flow over a continuously shrinking surface with mass suction was also investigated by Fang et al. [20]. Recently, Fang and Zhang [21] gave an exact solution of MHD boundary layer equations in closed analytical form for the flow over a shrinking sheet in the presence of suction at the surface. Hayat et al. [22] analyzed the MHD flow of a second grade fluid over a shrinking sheet. Steady two-dimensional and axisymmetric stagnationpoint flow with heat transfer on a shrinking sheet were investigated by Wang [23]. Mahapatra et al. [24] investigated the flow and heat transfer of the steady two-dimensional MHD stagnation-point flow of an electrically conducting incompressible viscous fluid over a shrinking sheet, the flow being permeated by a uniform transverse magnetic field. Note that with an added stagnation-point flow to contain the vorticity, similarity solution is possible even in the absence of suction at the surface. Recently, Fang et al. [25] obtained a closed form of analytical solution for the flows of nonNewtonian power-law fluids over a shrinking sheet with mass transfer.

Owing to wide range of technological applications, flow of non-Newtonian power-law fluid over a stretching/shrinking sheet has attracted many attention. To the best of author's knowledge, described the frequent mentioned literature, there is no investigation on the MHD stagnation point flow and radiative heat transfer of power-law fluid over a shrinking sheet. In this paper, we investigate the steady twodimensional MHD stagnation-point flow and heat transfer of an electrically conducting power-law fluid over a shrinking sheet, the flow being permeated by a uniform transverse magnetic field. Representative results for wall shear stress and velocity profiles are present for some values of the governing parameters. The temperature distribution in the flow is determined when the surface is held at a constant temperature, and the surface heat flux is also calculated. It is hoped that the obtained results will not only present useful

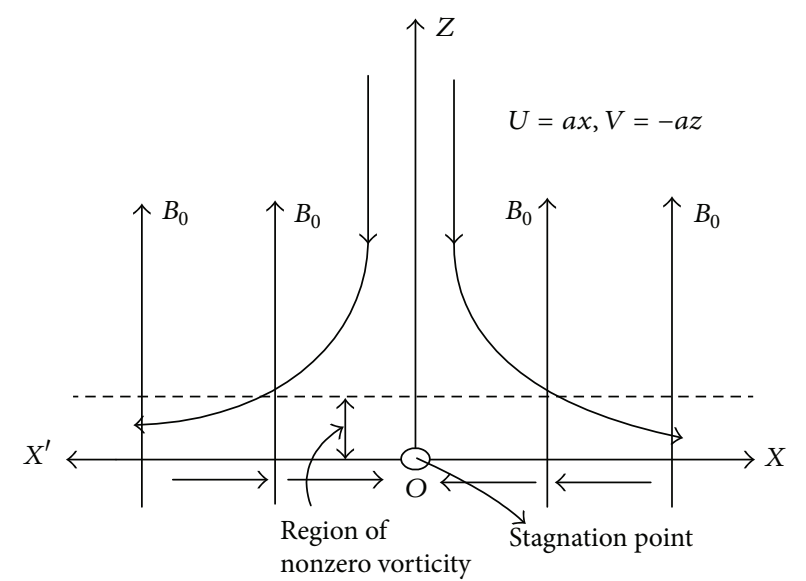

Figure 1: A physical model and the coordinate system.

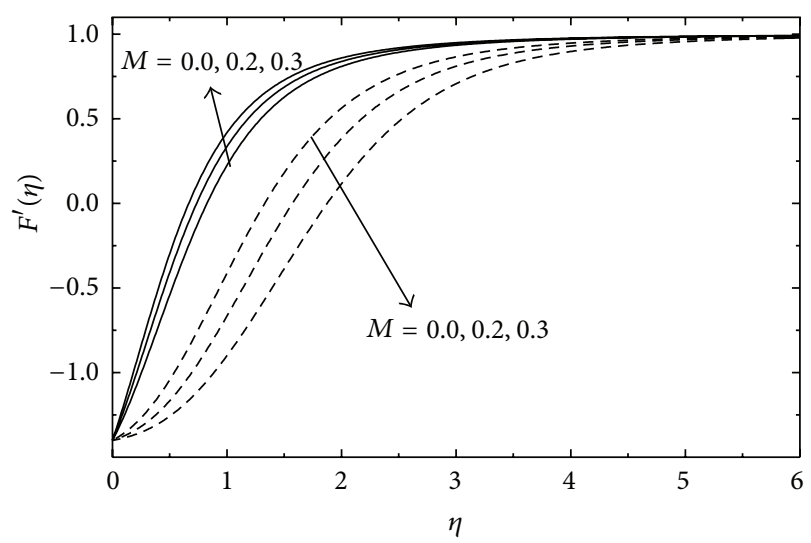

Figure 2: Variation of $F^{\prime}(\eta)$ with $\eta$ for several values of the magnetic parameter $M$ with $\alpha=-1.40$ and $n=0.4$ (solid line: first solution; dashed line: second solution).

information for applications but also serves as a complement to the previous studies.

\section{Flow Analysis}

Consider the steady two-dimensional stagnation-point flow of an electrically conducting incompressible power-law fluid towards a surface which is shrunk in its own plane with a velocity proportional to the distance from the stagnation point. Here $x$-axis runs along the shrinking surface in the direction opposite to sheet motion. A uniform magnetic field of strength $B_{0}$ is applied in a direction normal to the surface that is parallel to $z$-axis. We consider the two-dimensional irrotational stagnation-point flow at infinity given by $u=a x$, $w=-a z$, where $a(>0)$ is the strength of the stagnation-point flow. On the sheet, the velocities are $u=c x, w=0$, where $c(<0)$ is the shrinking rate (stretching rate if $c>0$ ). Here shrinking of the sheet is along the negative direction of $x$ axis. Figure 1 describes the physical model and the coordinate system, where the $x$ and $z$ axes are measured along the surface of the sheet and normal to it, respectively. 


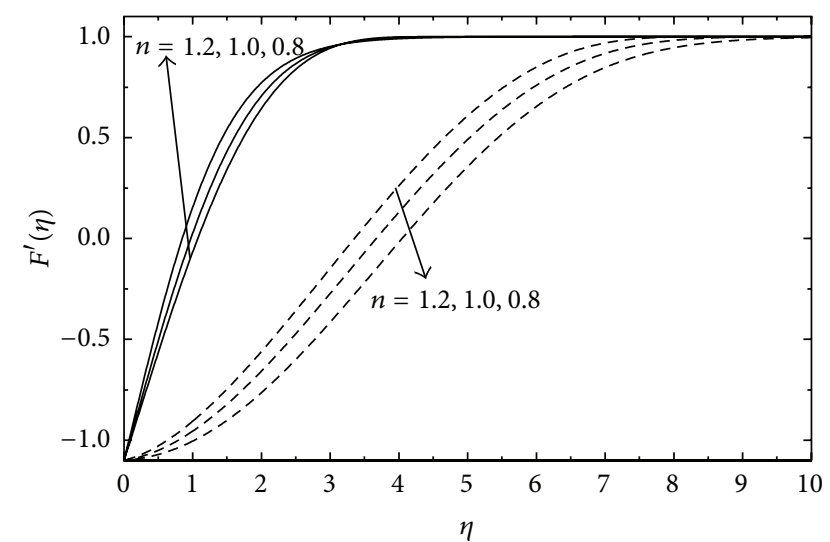

FIGURE 3: Variation of $F^{\prime}(\eta)$ with $\eta$ for several values of the powerlaw index $n$ with $\alpha=-1.2$ and $M=0.2$ (solid line: first solution; dashed line: second solution).

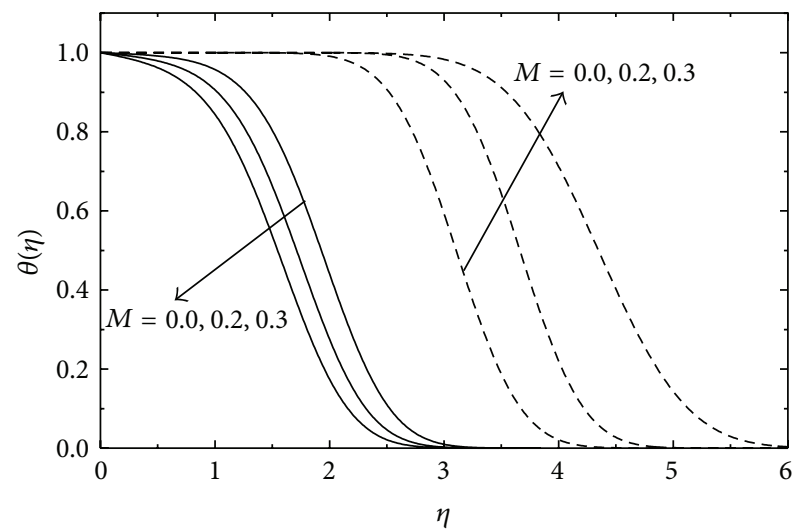

FIGURE 4: Variation of $\theta(\eta)$ with $\eta$ for several values of the magnetic parameter $M$ with $\alpha=-1.40, \operatorname{Pr}=10.0$, and $n=0.4$ (solid line: first solution; dashed line: second solution).

The rheological equation of state for an incompressible power-law fluid (Wilkinson [26]) is defined as

$$
\tau_{i j}=2 K\left|2 D_{m l} D_{m l}\right|^{(n-1) / 2} D_{i j},
$$

where $\tau_{i j}$ is the deviatoric part of the stress tensor $\sigma_{i j}=-p \delta_{i j}+$ $\tau_{i j}$. Here $p$ is the pressure, and $D_{i j}$ is the rate-of-strain tensor given by

$$
D_{i j}=\frac{1}{2}\left(\frac{\partial u_{i}}{\partial x_{j}}+\frac{\partial u_{j}}{\partial x_{i}}\right)
$$

where $u_{\mathrm{i}}$ is the velocity components. Here $K(>0)$ is called the consistency coefficient of the fluid, and $n(>0)$ is the powerlaw index. Fluids obeying constitutive equation (1) are called power-law fluids, and for such fluids the linear relationship between the stress tensor $\sigma_{i j}$ and the rate-of-strain tensor $D_{i j}$ does not hold. We note that for $n<1$, the fluid is called pseudoplastic power-law fluid; for $n>1$, it is called dilatant power-law fluid, and when $n=1$, the fluid is the Newtonian fluid.

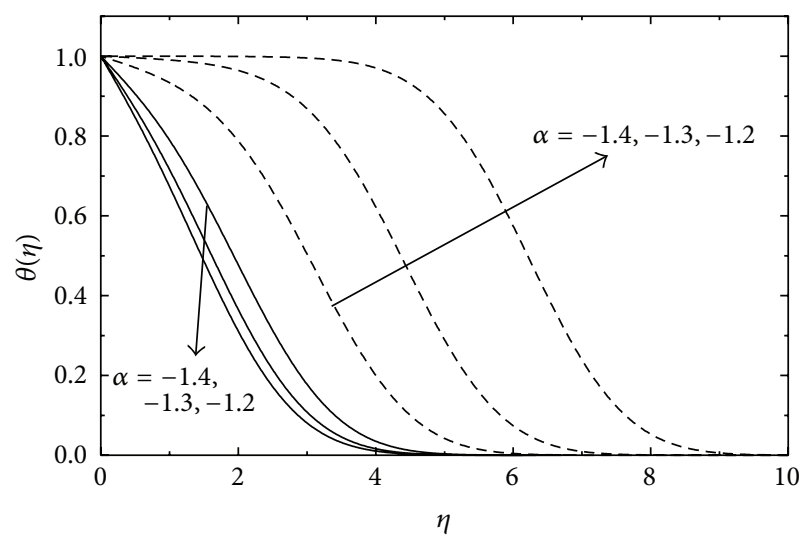

FIGURE 5: Variation of $\theta(\eta)$ with $\eta$ for several values of $\alpha(<0)$ with $M=0.2, \operatorname{Pr}=0.71$, and $n=0.4$ (solid line: first solution; dashed line: second solution).

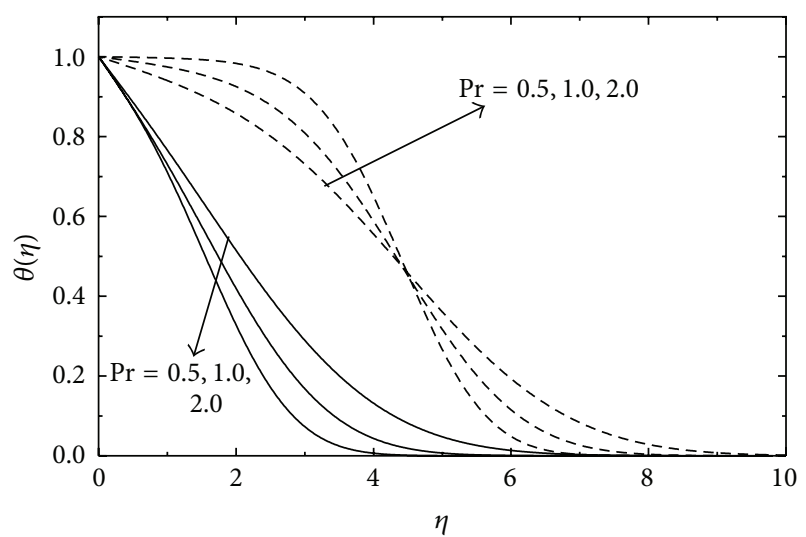

Figure 6: Variation of $\theta(\eta)$ with $\eta$ for several values of Pr with $M=$ $0.3, \alpha=-1.3$, and $n=0.4$ (solid line: first solution; dashed line: second solution).

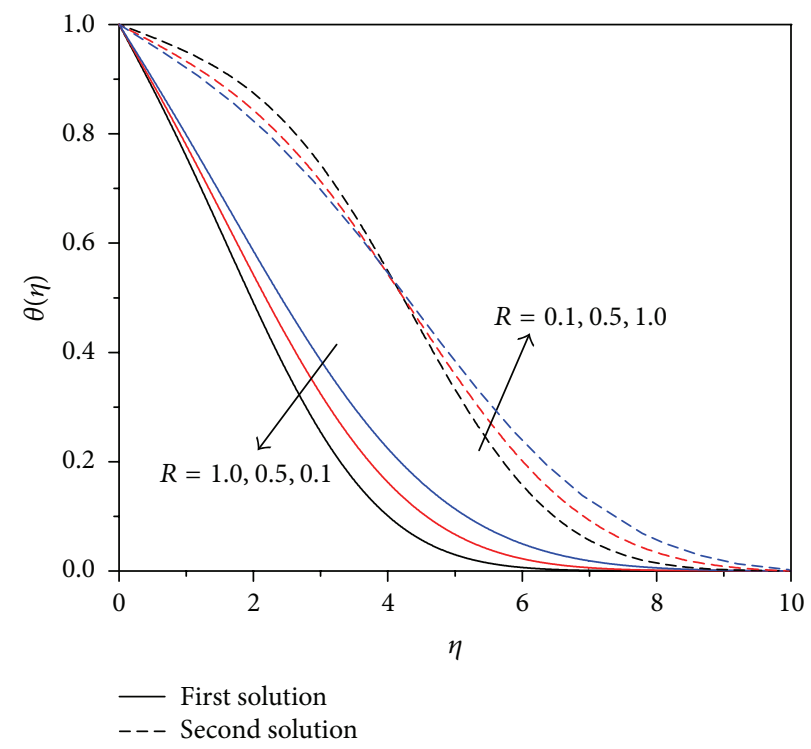

Figure 7: Variation of $\theta(\eta)$ with $\eta$ for several values of $R$ with $\alpha=$ $-1.4, M=0.3$, and $n=0.4$. 


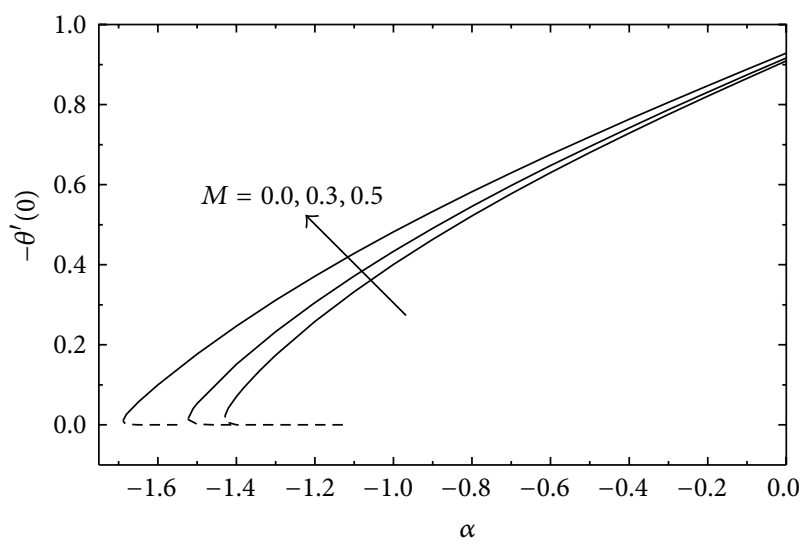

FIgURE 8: Variation of $-\theta^{\prime}(0)$ with $\alpha$ for several values of $M$ with $n=0.4$ and $\operatorname{Pr}=5.0$ (solid line: first solution; dashed line: second solution).

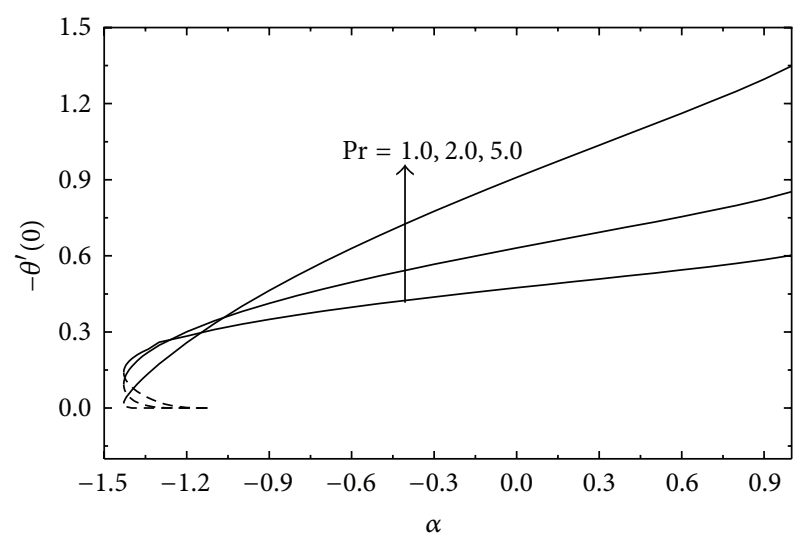

FIGURE 9: Variation of $-\theta^{\prime}(0)$ with $\alpha$ for several values of the Prandtl number $\operatorname{Pr}$ with $n=0.4$ and $M=0.2$ (solid line: first solution; dashed line: second solution).

Using boundary layer approximation, the equations for the steady MHD two-dimensional flow are in usual notation

$$
\begin{gathered}
u \frac{\partial u}{\partial x}+w \frac{\partial u}{\partial z}=-\frac{1}{\rho} \frac{\partial p}{\partial x}+\frac{1}{\rho} \frac{\partial \tau_{x z}}{\partial z}+\frac{1}{\rho}\left(\vec{j} \times \vec{B}_{0}\right)_{x} \\
0=\frac{\partial p}{\partial z}
\end{gathered}
$$

where $\rho$ is the density and $p$ is the pressure of the fluid. The last term in (3) stands for the $x$-component of the Lorentz force per unit mass where $\vec{j}$ is the electric current density and $\vec{B}_{0}$ is the imposed uniform magnetic field (acting along $z$-direction). In writing (3), we have neglected the induced magnetic field in comparison with the imposed magnetic field $B_{0}$ since the magnetic Reynolds number $R_{M}$ for the flow is assumed to be very small. Such an assumption is justified for flow of electrically conducting fluids such as liquid metals, for example, mercury and liquid Sodium (Shercliff [27]).
The equation of continuity is

$$
\frac{\partial u}{\partial x}+\frac{\partial w}{\partial z}=0
$$

Clearly the electric current in this two-dimensional flow acts parallel to the $y$-axis which is normal to $x z$-plane. So by Ohm's law

$$
j_{x}=0 \quad j_{y}=\sigma\left[E_{y}-u B_{0}\right], \quad j_{z}=0,
$$

where $\sigma$ is the electrical conductivity (assumed constant) of the fluid and $E_{y}$ is the electric field along $y$-axis. It is assumed that the electric current lines in this twodimensional configuration are closed in a manner consistent with the experiment. Now for the flow in the steady state, Maxwell's equation gives

$$
\vec{\nabla} \times \vec{E}=0,
$$

where $\vec{E}$ is the electric field which acts parallel to the $y$ direction. This gives $\partial E_{y} / \partial z=0$ and $\partial E_{y} / \partial x=0$, so that $E_{y}$ is a function of $y$ only.

Since the induced magnetic field is neglected in view of our assumption $R_{M} \ll 1$, electric current in the flow is determined from Ohm's law and not from $\vec{\nabla} \times \vec{B}=\mu_{e} \vec{j}, \mu_{e}$ being the magnetic permeability. But the consequence $\vec{\nabla} \cdot \vec{j}=$ 0 of this equation must be satisfied (Shercliff [27]). Since the physical variables are functions of $x$ and $z, \vec{\nabla} \cdot \vec{j}=0$ then gives from (6) that $E_{y}=$ constant. Then using (6), we find from (3) that

$$
u \frac{\partial u}{\partial x}+w \frac{\partial u}{\partial z}=-\frac{1}{\rho} \frac{\partial p}{\partial x}+\frac{1}{\rho} \frac{\partial \tau_{x z}}{\partial z}-\frac{\sigma B_{0}^{2} u}{\rho}+\frac{\sigma B_{0} E_{y}}{\rho} .
$$

Now since from (4), the pressure is independent of $z$, and the pressure gradient $\partial p / \partial x$ can now be obtained from (8) in the free stream as

$$
\frac{1}{\rho} \frac{\partial p}{\partial x}=-U(x) \frac{d U}{d x}-\frac{\sigma B_{0}^{2} U}{\rho}+\frac{\sigma B_{0} E_{y}}{\rho},
$$

where $U(x)$ is the free stream velocity and use is made of the fact $E_{y}=$ constant. Hence eliminating $\partial p / \partial x$ from (8) and (9), we get

$$
u \frac{\partial u}{\partial x}+w \frac{\partial u}{\partial z}=U \frac{d U}{d x}+\frac{1}{\rho} \frac{\partial \tau_{x z}}{\partial z}+\frac{\sigma B_{0}^{2}}{\rho}(U-u) .
$$

Note that the electric field does not directly affect the boundary layer equation, although it has an effect on the relation between the free stream velocity and the pressure distribution given by (9). Thus the governing equations for the velocity components $u$ and $w$ are (5) and (10). In the present problem we have $\partial u / \partial z>0$. Hence from (1) and (3), we have the shear stress as

$$
\tau_{x z}=K\left(\frac{\partial u}{\partial z}\right)^{n}
$$


Thus the momentum equation (10) reduces to the form

$$
u \frac{\partial u}{\partial x}+w \frac{\partial u}{\partial z}=U \frac{d U}{d x}+\frac{K}{\rho} \frac{\partial}{\partial z}\left(\frac{\partial u}{\partial z}\right)^{n}+\frac{\sigma B_{0}^{2}}{\rho}(U-u) .
$$

The boundary conditions are

$$
\begin{gathered}
u=c x, \quad w=0 \quad \text { at } z=0, \\
u \longrightarrow U(x)=a x, \quad w \longrightarrow-a z \quad \text { as } z \longrightarrow \infty,
\end{gathered}
$$

where $c>0$ is for stretching and $c<0$ is for shrinking. We introduce the similarity transformations

$$
\begin{gathered}
\psi=\left(\frac{K / \rho}{a^{1-2 n}}\right)^{1 /(n+1)} x^{2 n /(n+1)} F(\eta), \\
\eta=z\left(\frac{a^{2-n}}{K / \rho}\right)^{1 /(n+1)} x^{(1-n) /(1+n)},
\end{gathered}
$$

where the stream function $\psi$ is defined by the following relations:

$$
u=\frac{\partial \psi}{\partial z}, \quad w=-\frac{\partial \psi}{\partial x} .
$$

With the values of $u$ and $w$ obtained from (14)-(15), we find that the equation of continuity (5) is identically satisfied. Substituting (14)-(15) into (12), we obtain the following nonlinear ordinary differential equation:

$$
\begin{gathered}
n\left[F^{\prime \prime}(\eta)\right]^{(n-1)} F^{\prime \prime \prime}(\eta)+\left(\frac{2 n}{n+1}\right) F(\eta) F^{\prime \prime}(\eta) \\
-F^{\prime^{2}}(\eta)+1+M^{2}\left(1-F^{\prime}(\eta)\right)=0,
\end{gathered}
$$

where $M\left(=B_{0}(\sigma / \rho a)^{1 / 2}\right)$ is the dimensionless magnetic parameter and a prime denotes differentiation with respect to the independent variable $\eta$. From (13), we get the dimensionless boundary conditions as

$$
F(0)=0, \quad F^{\prime}(0)=\frac{c}{a}=\alpha, \quad F^{\prime}(\infty)=1 .
$$

Equation (16) along with the boundary conditions (17) holds for both $\alpha>0$ (i.e., for stretching sheet) and $\alpha<0$ (i.e., for shrinking sheet). The skin friction coefficient $C_{f}$ at the wall is given by

$$
\begin{aligned}
C_{f} & =\frac{\tau_{w}}{(1 / 2) \rho(c x)^{2}} \\
& =2\left[F^{\prime \prime}(0)\right]^{n}\left[\frac{(c x)^{2-n} x^{n}}{K / \rho}\right]^{-1 /(1+n)} \\
& =2\left[F^{\prime \prime}(0)\right]^{n}\left(\operatorname{Re}_{x}\right)^{-1 /(1+n)}
\end{aligned}
$$

where $\operatorname{Re}_{x}=(c x)^{2-n} x^{n} /(K / \rho)$ is the local Reynolds number based on the sheet velocity $c x$.

\section{Heat Transfer}

To determine the temperature distribution in the above flow field, we solve the following energy equation using boundary layer approximation, neglecting viscous and ohmic dissipation,

$$
u \frac{\partial T}{\partial x}+w \frac{\partial T}{\partial z}=\kappa \frac{\partial^{2} T}{\partial z^{2}}-\frac{1}{\rho c_{p}} \frac{\partial q_{r}}{\partial z},
$$

where $\kappa, c_{p}$, and $T$ denote the thermal diffusivity, the specific heat at constant pressure, and the temperature of the fluid, respectively, and $q_{r}$ is the radiative heat flux. Using Rosseland's approximation for radiation (Brewster [28]), we can write

$$
q_{r}=-\frac{4 \sigma^{*}}{3 K^{*}} \frac{\partial T^{4}}{\partial z}
$$

where $\sigma^{*}$ is the Stefan-Boltzmann constant and $K^{*}$ is the mean absorption coefficient. Assuming the temperature difference within the flow is such that $T^{4}$ may be expanded in a Taylor series about $T_{\infty}$ and neglecting higher order terms, we get $T^{4} \approx 4 T_{\infty}^{3} T-3 T_{\infty}^{4}$. Hence (19) becomes

$$
u \frac{\partial T}{\partial x}+w \frac{\partial T}{\partial z}=\kappa \frac{\partial^{2} T}{\partial z^{2}}+\frac{16 \sigma^{*} T_{\infty}^{3}}{3 K^{*} \rho c_{p}} \frac{\partial^{2} T}{\partial z^{2}} .
$$

The appropriate boundary conditions are

$$
T=T_{w} \quad \text { at } z=0, \quad T=T_{\infty} \quad \text { as } z \longrightarrow \infty,
$$

where $T_{w}$ and $T_{\infty}$ are constants with $T_{w}>T_{\infty}$. We introduce the dimensionless temperature $\theta$ as

$$
\theta(\eta)=\frac{T-T_{\infty}}{T_{w}-T_{\infty}}
$$

Now the energy equation (19), with the above boundary condition, becomes

$$
\left(1+\frac{4 R}{3}\right) \theta^{\prime \prime}(\eta)+\frac{2 n}{n+1} \operatorname{Pr} F(\eta) \theta^{\prime}(\eta)=0,
$$

where $\operatorname{Pr}=(c / \kappa)\left((K / \rho) / c^{2-n}\right) \operatorname{Re}_{x}^{(n-1) /(n+1)}$ is the generalized Prandtl number and $R=4 \sigma^{*} T_{\infty}^{3} / \kappa K^{*} \rho c_{p}$ is the thermal radiation parameter. The boundary conditions for $\theta$ are obtained from (22) and (23) as

$$
\theta(0)=1, \quad \theta(\infty)=0 .
$$

It is interesting to note that when $\alpha=1$ (i.e., $a=c$ ), then (16) together with the boundary conditions (17) admits of an exact analytical solution $F(\eta)=\eta$. Hence in this case, the energy equation (24) reduces to the form

$$
\theta^{\prime \prime}(\eta)+k \eta \theta^{\prime}(\eta)=0
$$

where $k=(2 n /(n+1)) \operatorname{Pr} /(1+4 R / 3)$ is a positive constant. Solution of (26) can be written as

$$
\theta(\eta)=C_{1} \sqrt{\frac{\pi}{2 k}} \operatorname{erf}\left(\sqrt{\frac{k}{2} \eta}\right)+C_{2}
$$


TABLE 1: Comparison of the values of $F^{\prime \prime}(0)$ (with $n=1$ and $M=0$ ) for stretching sheet with different values of $\alpha(>0)$.

\begin{tabular}{lcc}
\hline$\alpha$ & Present study & Wang [23] \\
\hline 0.0 & 1.232588 & 1.232588 \\
0.1 & 1.146560 & 1.146560 \\
0.2 & 1.051131 & 1.051130 \\
0.5 & 0.713296 & 0.713300 \\
1.0 & 0 & 0 \\
2.0 & -1.887308 & -1.88731 \\
5.0 & -10.264751 & -10.26475 \\
\hline
\end{tabular}

where $C_{1}$ and $C_{2}$ are two constants of integration and erf denotes the error function. Using the boundary conditions (25), we have the solution as

$$
\theta(\eta)=1-\operatorname{erf}\left(\sqrt{\frac{k}{2} \eta}\right) .
$$

\section{Numerical Solutions}

The governing momentum equation (16) along with the boundary conditions (17) and the thermal equation (24) together with the boundary conditions (25) are solved numerically. To do this, we first transform the nonlinear differential equation (16) to a system of three first-order differential equations, which are solved by means of a standard fourth-order Runge-Kutta scheme coupled with a conventional shooting procedure. The step size $\eta=0.001$ and the convergence criteria $10^{-6}$ are used in this program. In practice, the value $\eta=\infty$ must be replaced by an approximation $\eta=\eta_{\max }$, where the value of $\eta_{\max }$ is chosen; in such a way, all profiles satisfy the far field boundary conditions asymptotically. We ran our bulk computations with the value $\eta_{\max }=12$, which was sufficient to achieve the far field boundary conditions asymptotically for all values of the parameters considered. Then a Newton iteration procedure is employed to assure quadratic convergence of the iterations to satisfy the boundary condition $F^{\prime}(\infty)=1$.

Once the numerical values of $F(\eta)$ are known, we have solved the thermal equation (24) together with the boundary condition (25) using the same technique for obtaining the numerical values of $\theta(\eta)$. An explanation of this algorithm is given in Gladwell and Sayers [29].

\section{Results and Discussion}

In order to validate the method used in this study and to judge the accuracy of the present analysis, comparison with the results of Wang [23] for the skin friction coefficient $F^{\prime \prime}(0)$ for Newtonian fluid (taking $n=1$ ) in the absence of magnetic field $(M=0)$ for stagnation point flow towards a stretching/shrinking sheet is made and found excellent agreement, as shown in Tables 1 and 2. Therefore we are confident that the present results are accurate.

From a physical point of view, vorticity generated at the shrinking sheet is not confined within a boundary layer, and
TABLE 2: Comparison of our results in the case $n=1$ and $M=0$ with Wang's [23] results for $F^{\prime \prime}(0)$ in the shrinking case.

\begin{tabular}{lcccc}
\hline & \multicolumn{2}{c}{ Wang [23] } & \multicolumn{2}{c}{ Present work } \\
$\alpha$ & $\begin{array}{c}\text { First } \\
\text { solution }\end{array}$ & $\begin{array}{c}\text { Second } \\
\text { solution }\end{array}$ & $\begin{array}{c}\text { First } \\
\text { solution }\end{array}$ & $\begin{array}{c}\text { Second } \\
\text { solution }\end{array}$ \\
\hline-0.25 & 1.40224 & - & 1.402242 & - \\
-0.50 & 1.49567 & - & 1.495672 & - \\
-0.75 & 1.48930 & - & 1.489296 & - \\
-1.00 & 1.32882 & 0.0 & 1.328819 & 0.0 \\
-1.10 & - & - & 1.186680 & 0.049229 \\
-1.15 & 1.08223 & 0.116702 & 1.082232 & 0.116702 \\
-1.20 & - & - & 0.932470 & 0.233648 \\
-1.2465 & 0.55430 & - & 0.584374 & 0.554215 \\
\hline
\end{tabular}

a steady solution is not possible unless sufficient stagnation flow is added to the free stream. Even with an added stagnation flow, a steady solution is possible only when the ratio of shrinking velocity and free stream velocity (i.e., $\alpha$ ) becomes less than a certain numerical value which again depends on the magnetic parameter $(M)$ and the powerlaw index parameter $(n)$. Note that $(16)$ is nonlinear. It may be possible to have multiple or bifurcated solutions. Our numerical results reveal that for Newtonian fluid (i.e., $n=1$ ), the solution of (16) satisfying boundary conditions (17) is unique for $\alpha>-1.0$. But dual solutions exist for $-1.2465 \leq$ $\alpha \leq-1.00$, and no similarity solution is found for $\alpha<$ -1.2465 . These results agree well with those of (Wang [23]) who studied steady stagnation-point flow over a shrinking sheet. The ranges of $\alpha$ for unique solution, dual solutions, and nonexistence of the solution for different values of $M$ and $n$ are present in concise form in Table 3 . From Table 3 it is seen that as the strength of the magnetic parameter $M$ increases, the range of $\alpha$ where similarity solutions exist gradually increases. The novel result which emerges from the above analysis is that the range of $\alpha$ where dual solutions exist progressively decreases with increase in $M$. It is also observed that as the power-law index $n$ increases, the range of $\alpha$ where two solutions exist decreases.

Figure 2 shows the variation of $F^{\prime}(\eta)$ and the horizontal component of velocity, with $\eta$ for several values of the magnetic parameter $M$ with fixed values of $n(=0.4)$ and $\alpha(=-1.40)$ for the first and second solution branches. In the following discussion, we identify the first solution branch as the solution with higher values of $F^{\prime \prime}(0)$ for a given $\alpha$ and second solution branch as the lesser values of $F^{\prime \prime}(0)$ for the same $\alpha$ (in the dual solution range). It is observed that for the first solution branch, the horizontal velocity component at a point increases with increase in $M$, but for the second solution branch it decreases with increase in $M$. Figure 3 shows the variation of $F^{\prime}(\eta)$ with $\eta$ for different values of the power-law index $n$ for fixed value of the shrinking rate parameter $\alpha(=-1.2)$ and $M=0.2$ for both the solution branches. The figure shows that $\left|F^{\prime}(\eta)\right|$ decreases with increase in $n$ for the first solution branch, but for the second solution branch it increases with increase in $n$. 
TABLE 3: Ranges of $\alpha$ for unique solution, dual solutions, and nonexistence of the solution for different values of $M$ and $n$.

\begin{tabular}{ccccc}
\hline$n$ & $M$ & Unique solution & Dual solution & Nonexistence of solution \\
\hline \multirow{2}{*}{0.4} & 0.0 & $\alpha>-1.1291$ & $-1.42890 \leq \alpha \leq-1.1291$ & $\alpha<-1.4289$ \\
& 0.3 & $\alpha>-1.2850$ & $-1.52221 \leq \alpha \leq-1.28501$ & $\alpha<-1.52221$ \\
1.0 & 0.0 & $\alpha>-1.0000$ & $-1.2465 \leq \alpha \leq-1.00000$ & $\alpha<-1.2465$ \\
& 0.3 & $\alpha>-1.18300$ & $-1.32604 \leq \alpha \leq-1.18300$ & $\alpha<-1.32604$ \\
1.5 & 0.0 & $\alpha>-1.0369$ & $-1.18291 \leq \alpha \leq-1.0369$ & $\alpha<-1.18291$ \\
& 0.3 & $\alpha>-1.1340$ & $-1.25776 \leq \alpha \leq-1.13401$ & $\alpha<-1.25776$ \\
\hline
\end{tabular}

Figure 4 displays the variation of $\theta(\eta)$ with $\eta$ for different values of $M$ for both the solution branches with fixed values of $\alpha(=-1.40), \operatorname{Pr}(=10.0)$, and $n=0.4$. It is observed that temperature at a point decreases with increase in $M$ for the first solution branch and temperature at a point increases with increase in $M$ for the second solution branch. From a physical point of view, this follows from the fact that as the magnetic parameter $M$ increases, the boundary layer becomes thicker for the first branch, while it becomes thinner for the second branch. Figure 5 displays the variation of $\theta(\eta)$ with $\eta$ for several values of $\alpha(<0)$ with fixed values of $M=$ $0.2, \operatorname{Pr}(=0.71)$, and $n(=0.4)$ for the first and the second solution branches. The figure reveals that temperature at a point increases with increase in $|\alpha|$ for the first branch and decreases with increase in $|\alpha|$ for the second branch. From a physical point of view, this follows from the fact that the boundary layer becomes thicker as $|\alpha|$ increases for the first branch and it becomes thinner for the second branch as $|\alpha|$ increases.

Figure 6 shows the variation of $\theta(\eta)$ with $\eta$ for several values of $\operatorname{Pr}$ with fixed values of $M=0.3, \alpha(=-1.3)$, and $n(=0.4)$ for both the solution branches. The figure indicates that as Pr increases, temperature at a point decreases for the first solution branch. For the second branch temperature at a point increases with increase in $\mathrm{Pr}$ in a certain neighbourhood of the shrinking sheet but beyond this neighbourhood temperature at a point decreases with increase in Pr. Figure 7 shows the variation of temperature profiles for various values of the radiation parameter $R$ for fixed values of the other parameters. The figure reveals that an increase in radiation parameter leads to an increase in the temperature at a point for both the solution branches except in a small region (in case of second solution).

Figure 8 displays the variation of the dimensionless surface heat flux $-\theta^{\prime}(0)$ with $\alpha(<0)$ for different values of the magnetic parameter $M$. The figure reveals that for fixed values of $\alpha$ and $\operatorname{Pr}$, the surface heat flux $-\theta^{\prime}(0)$ increases with increase in $M$ for the first solution branch. Interestingly for the second solution branch for the same values of $\alpha$ and $\mathrm{Pr}$, the surface heat flux $-\theta^{\prime}(0)$ is almost zero for different values of $M$. Figure 9 shows the variation of the dimensionless surface heat flux $-\theta^{\prime}(0)$ with $\alpha$ for different values of $\operatorname{Pr}$ with fixed $n(=0.4)$ and $M=0.3$. It is seen that up to certain value of $\alpha$, $-\theta^{\prime}(0)$ increases with increase in $\operatorname{Pr}$, and beyond this $\alpha,-\theta^{\prime}(0)$ decreases with increase in $\operatorname{Pr}$ for the first solution branch. Again for the second solution branch, $-\theta^{\prime}(0)$ decreases as $\mathrm{Pr}$ increases. It is to be noted that for higher values of Prandtl number, $-\theta^{\prime}(0)$ is almost zero for the second solution branch.

\section{Conclusion}

The present paper deals with the analysis of the steady twodimensional MHD stagnation-point flow and heat transfer of a power-law fluid over a continuously shrinking surface in the presence of thermal radiation. In comparison to the stretching sheet, the shrinking sheet has some interesting characteristics. Greatly different solution behaviour with multiple solution branches (for certain value of the shrinking rate parameter) is found compared with the corresponding stretching sheet problem. The following conclusions can be drawn from the numerical results obtained.

(i) As the strength of the magnetic parameter $M$ increases, the range of $\alpha$ where similarity solutions exist gradually increases.

(ii) Dual solutions exist for certain parameter domain that depends on $n, M$, and $\alpha$.

(iii) Velocity at a point increases with increase in $M$ for the first solution, and it decreases with increase in $M$ for the second solution.

(iv) Temperature at a point decreases with increase in $M$ for the first solution and increases with increase in $M$ for the second solution.

(v) An increase in radiation parameter leads to an increase in the temperature at a point.

\section{Acknowledgment}

The author appreciates the comments of the reviewer, which have led to improvement of the paper.

\section{References}

[1] A. B. Metzner, "Heat transfer in non-Newtonian fluids," Advances in Heat Transfer, vol. 2, pp. 357-397, 1965.

[2] K. Hiemenz, "Die Grenzschicht in Einem in Dem Gleichformingen Flussigkeitsstrom Eingetauchten Gerade Kreiszlinder," Dingler's Polytechnic Journal, vol. 326, pp. 321-410, 1911.

[3] F. Homann, "Der Einfluss Grosser Zahighkeit Bei Der Stromung um den Zylinder und um die Kugel," Zeitschrift für Angewandte Mathematik und Mechanik, vol. 16, pp. 153-164, 1936.

[4] L. J. Crane, "Flow past a stretching plate," Zeitschrift für Angewandte Mathematik und Physik, vol. 21, pp. 645-647, 1970.

[5] K. B. Pavlov, "Magnetohydrodynamic flow of an incompressible viscous fluid caused by the deformation of a plane surface," Magnitnaya Gidrodinamika, vol. 4, pp. 146-147, 1974. 
[6] H. I. Andersson, "MHD flow of a viscoelastic fluid past a stretching surface," Acta Mechanica, vol. 95, no. 1-4, pp. 227230, 1992.

[7] H. I. Andersson and B. S. Dandapat, "Flow of a power-law fluid over a stretching sheet," Stability and Applied Analysis of Continuous Media, vol. 1, pp. 339-347, 1991.

[8] H. I. Andersson, K. H. Bech, and B. S. Dandapat, "Magnetohydrodynamic flow of a power-law fluid over a stretching sheet," International Journal of Non-Linear Mechanics, vol. 27, no. 6, pp. 929-936, 1992.

[9] T. C. Chiam, "Stagnation-point flow towards a stretching plate," Journal of the Physical Society of Japan, vol. 63, no. 6, pp. 24432444, 1994.

[10] K. Vajravelu, "Flow and heat transfer in a saturated porous medium over a porous medium over a stretching," Zeitschrift für Angewandte Mathematik und Mechanik, vol. 74, pp. 605-614, 1994.

[11] K. Vajravelu and J. Nayfeh, "Convective heat transfer at a stretching sheet," Acta Mechanica, vol. 96, no. 1-4, pp. 47-54, 1993.

[12] T. R. Mahapatra and A. S. Gupta, "Heat transfer in stagnationpoint flow towards a stretching sheet," Heat and Mass Transfer, vol. 38, no. 6, pp. 517-521, 2002.

[13] S. Abel, K. V. Prasad, and A. Mahaboob, "Buoyancy force and thermal radiation effects in MHD boundary layer viscoelastic fluid flow over continuously moving stretching surface," International Journal of Thermal Sciences, vol. 44, no. 5, pp. 465476, 2005.

[14] T. R. Mahapatra, S. K. Nandy, and A. S. Gupta, "Magnetohydrodynamic stagnation-point flow of a power-law fluid towards a stretching surface," International Journal of NonLinear Mechanics, vol. 44, no. 2, pp. 123-128, 2009.

[15] K. V. Prasad, D. Pal, and P. S. Datti, "MHD power-law fluid flow and heat transfer over a non-isothermal stretching sheet," Communications in Nonlinear Science and Numerical Simulation, vol. 14, no. 5, pp. 2178-2189, 2009.

[16] K. V. Prasad and K. Vajravelu, "Heat transfer in the MHD flow of a power law fluid over a non-isothermal stretching sheet," International Journal of Heat and Mass Transfer, vol. 52, no. 2122, pp. 4956-4965, 2009.

[17] S. Goldstein, "On backward boundary layers and flow in converging passages," Journal of Fluid Mechanics, vol. 21, no. 1, pp. 33-45, 1965.

[18] M. Miklavcic and C. Y. Wang, "Viscous flow due to a shrinking sheet," Quarterly of Applied Mathematics, vol. 64, no. 2, pp. 283290, 2006.

[19] T. Fang, "Boundary layer flow over a shrinking sheet with power-law velocity," International Journal of Heat and Mass Transfer, vol. 51, no. 25-26, pp. 5838-5843, 2008.

[20] T. Fang, J. Zhang, and S. Yao, "Viscous flow over an unsteady shrinking sheet with mass transfer," Chinese Physics Letters, vol. 26, no. 1, Article ID 014703, 2009.

[21] T. Fang and J. Zhang, "Closed-form exact solutions of MHD viscous flow over a shrinking sheet," Communications in Nonlinear Science and Numerical Simulation, vol. 14, no. 7, pp. 2853-2857, 2009.

[22] T. Hayat, Z. Abbas, and M. Sajid, "On the analytic solution of magnetohydrodynamic flow of a second grade fluid over a shrinking sheet," Journal of Applied Mechanics, vol. 74, no. 6, pp. 1165-1171, 2007.
[23] C. Y. Wang, "Stagnation flow towards a shrinking sheet," International Journal of Non-Linear Mechanics, vol. 43, no. 5, pp. 377-382, 2008.

[24] T. R. Mahapatra, S. K. Nandy, and A. S. Gupta, "Momentum and heat transfer in MHD stagnation-point flow over a shrinking sheet," Journal of Applied Mechanics, vol. 78, no. 2, Article ID 021015, 2011.

[25] T. Fang, H. Tao, and Y. Zhong, "Non-Newtonian power-law flud flow over a shrinking sheet," Chinese Physics Letters, vol. 29, no. 11, Article ID 114703, 2012.

[26] W. L. Wilkinson, Non-Newtonian Fluids, Pergamon Press, London, UK, 1960.

[27] J. A. Shercliff, A Textbook of Magnetohydrodynamics, Pergamon Press, Oxford, UK, 1965.

[28] M. Q. Brewster, Thermal Radiative Transfer Properties, John Wiley \& Sons, New York, NY, USA, 1972.

[29] I. Gladwell and D. K. Sayers, Computational Techniques for Ordinary Differential Equations, Academic Press, London, UK, 1980. 


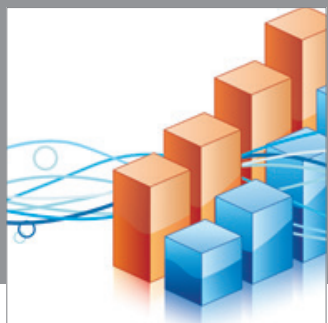

Advances in

Operations Research

mansans

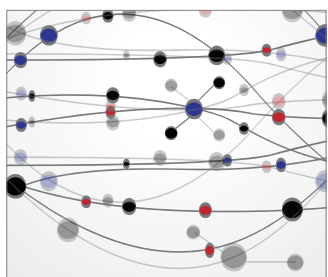

The Scientific World Journal
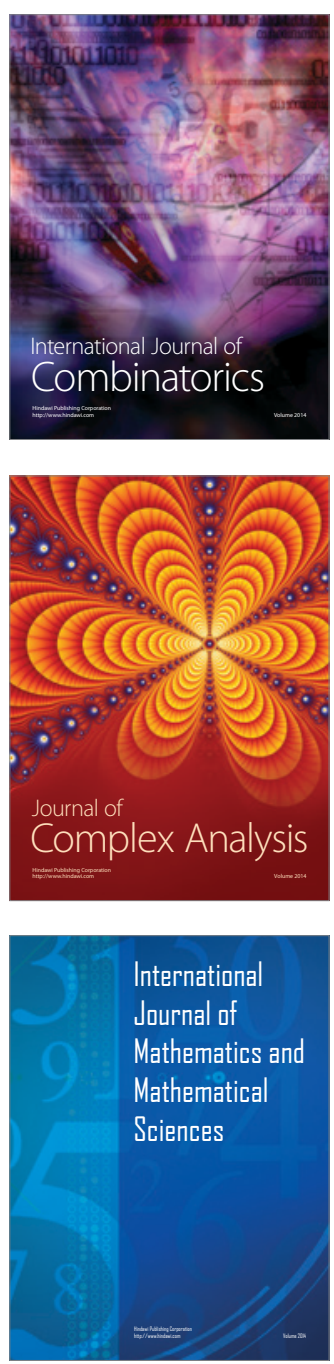
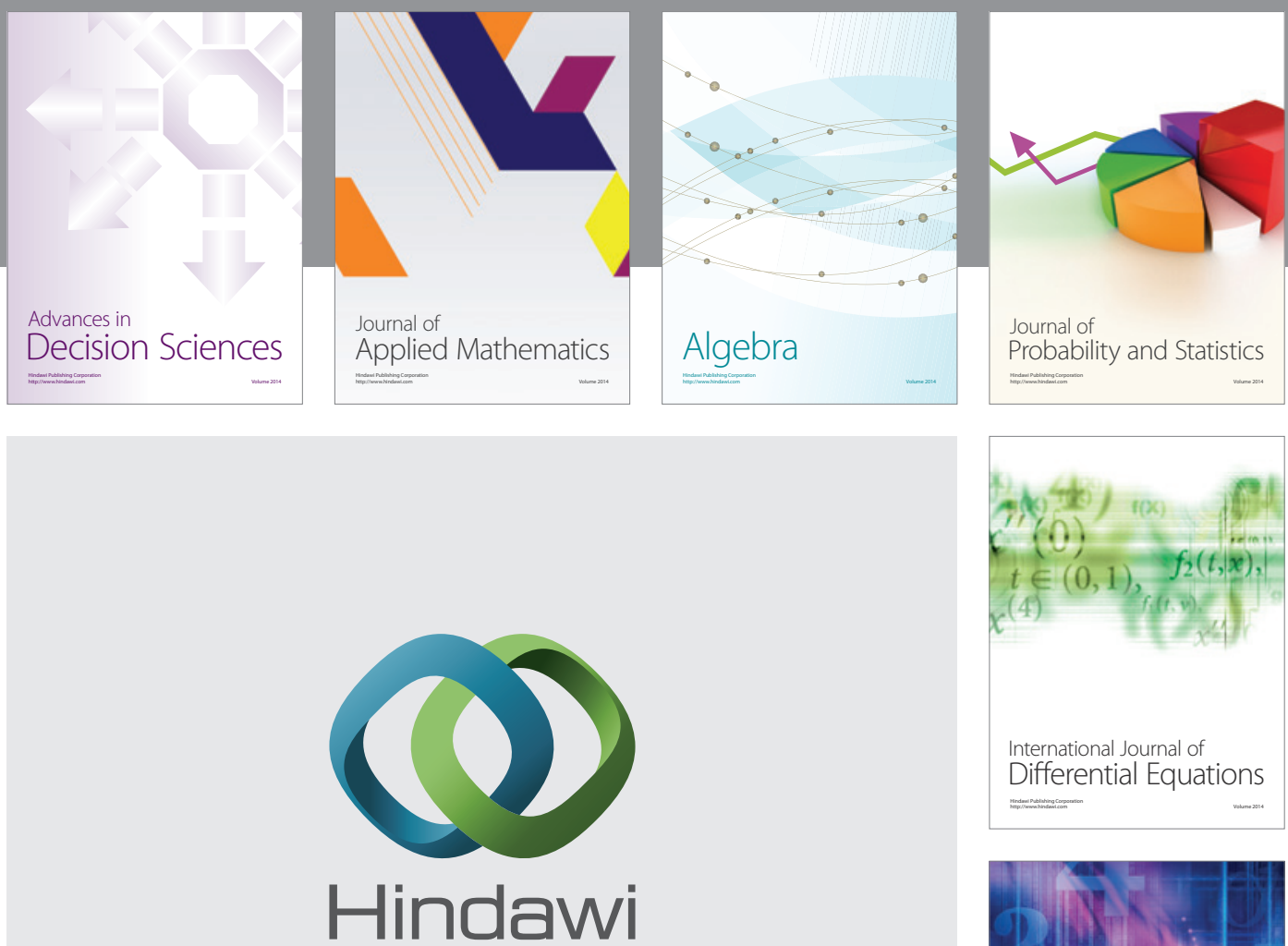

Submit your manuscripts at http://www.hindawi.com
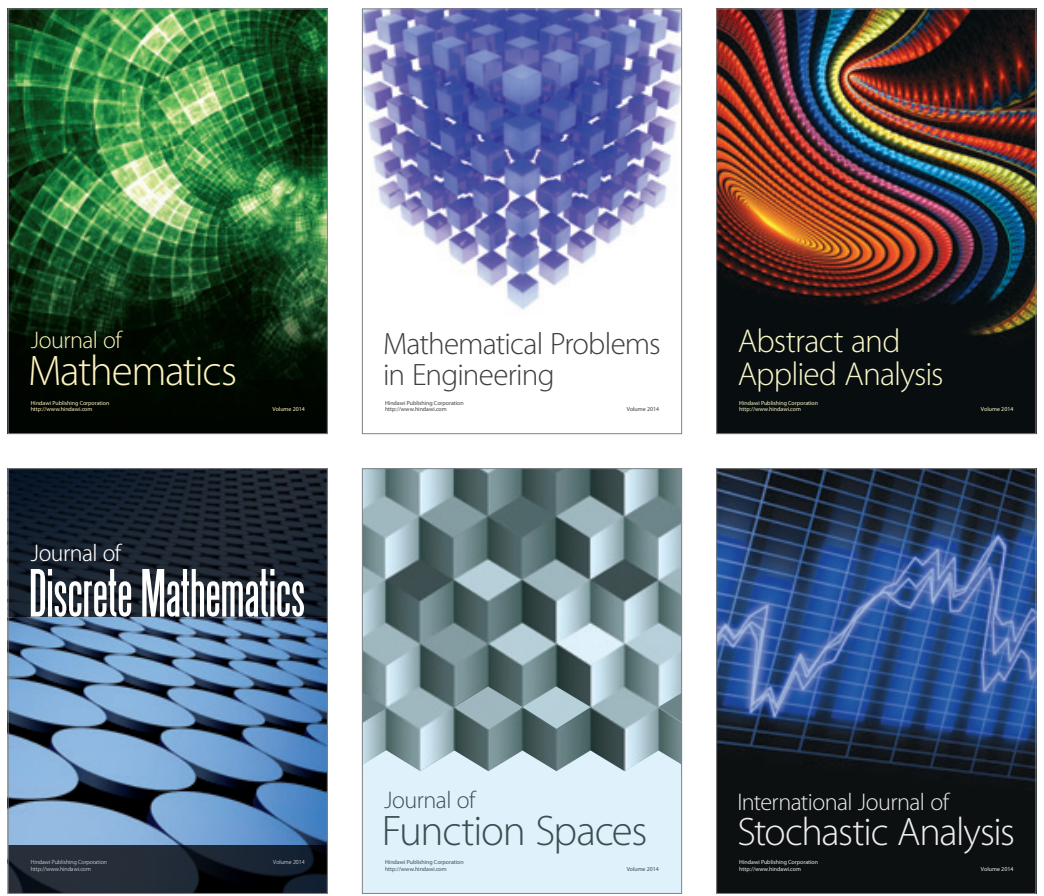

Journal of

Function Spaces

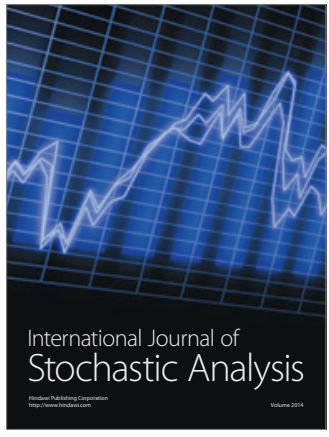

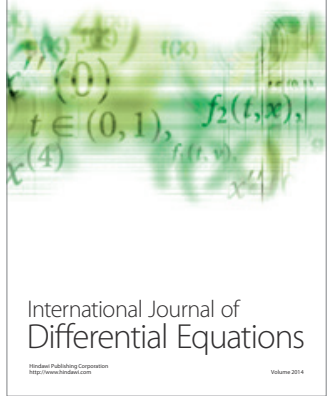
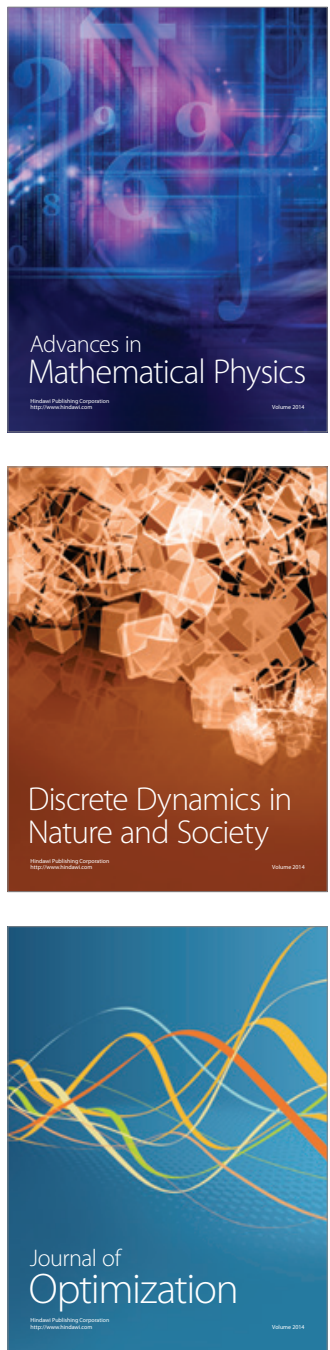